\title{
Electrical Conductivity of PEDOT:PSS Film Prepared through Organic Compound Addition
}

\author{
M. A. Guziak, T. Nishizaki, Y. Honma, K. Watanabe and T. Sasaki \\ Institute for Materials Research, Tohoku University, Katahira 2-1-1, Sendai 980-8577, Japan \\ Fax: +81-22-215-2026, e-mail: mguziak@imr.tohoku.ac.jp
}

We report on the method of enhancement of the electrical conductivity of poly(3,4-ethylene dioxythiophene):poly(4-styrenesulfonate) (PEDOT:PSS) films through a treatment with newly found organic additives, such as ethylene carbonate (EC) and ethylene sulfite (ES). Addition of these secondary dopants to PEDOT:PSS aqueous solution and subsequent film preparation resulted in conductivity enhancement by three orders of magnitude. Electrical resistivity of EC $(3.1 \mathrm{~m} \Omega \mathrm{cm})$ and ES $(2.9 \mathrm{~m} \Omega \mathrm{cm})$ added PEDOT:PSS films are comparable with previously reported secondary dopant, such as dimethyl sulfoxide (DMSO) (2.8 $\mathrm{m} \Omega \mathrm{cm})$. The temperature dependence of electrical resistivity of EC, ES and DMSO added PEDOT:PSS films indicates 3D-varible range hopping (VRH) conduction. Improving the electrical conductivity, however, we need to consider a crossover from VRH to metallic conduction at low temperature.

Keywords: PEDOT:PSS, secondary dopant, variable range hopping, electrical resistivity

\section{INTRODUCTION}

Poly(3,4-ethylenedioxythiophene) doped with poly(4-styrenesulfonate) (PEDOT:PSS) has become one of the significant conductive organic polymers in a last few decades. Due to its high electrical conductivity, visible light transparency, low cost processing and flexibility it has been used commercially in developing variety of technically useful industrial products such as solid electrolyte capacitors and antistatic coatings [1] Since the high electrical conductivity is required for many of these applications, intensive studies and various approaches have been undertaken to improve its conductivity: chemical modification of the EDOT, hence PEDOT [2]; preparation method [3]; solvent posttreatment [4] and secondary dopant addition reported first by MacDiarmid and Epstein [5]. The term "secondary doping" is defined as a substance causing a further increase in conductivity of a primarily doped conjugated polymer by up to several orders of magnitude. Contrary to "primary doping", the "secondary doping" has irreversible effect and results in permanency of improved conductivity even upon its complete removal from the prepared film. Although several mechanisms for conductivity improvement have been proposed, its physical origin is still poorly understood. However, recent structural model shows existence of nano-crystalline PEDOT grown in ethylene glycol post-treated PEDOT:PSS film [6]. In addition, the conductivity enhancement by using polar solvent closely relates to the size of PEDOT nano-crystals. Such improvement of the crystallinity of PEDOT is considered to occur during evaporation process of both solvent and water. Therefore, it is essential to investigate the effect of other agents of different structural nature on conductive properties and mechanism of charge transport of PEDOT:PSS film.

In this study, we report on the effect of EC and ES added PEDOT:PSS film on charge transport properties. We observe pronounced decrease of room temperature DC resistivity of the system: from $44 \Omega \mathrm{cm}$ ( $\mathrm{PH}$ grade) to 8.3, 7.2, $3.8 \mathrm{~m} \Omega \mathrm{cm}$ and from $0.8 \Omega \mathrm{cm}$ (PH1000) to 3.1, 2.9 and $2.8 \mathrm{~m} \Omega \mathrm{cm}$ by the change of solvent for the EC, ES and DMSO respectively.

\section{EXPERIMENTAL}

\subsection{Materials}

The PEDOT:PSS aqueous dispersion (grades Clevios $^{\mathrm{TM}} \mathrm{PH}$ and $\left.\mathrm{PH} 1000\right)$ was purchased from Heraeus Holding $\mathrm{GmbH}$ with solid content of 1.2-1.4\% and $1.0-1.3 \%$, respectively. For the preparation of PEDOT:PSS/organic compound solutions, following solvents were used: ethylene carbonate (EC), ethylene 
sulfite (ES) and dimethyl sulfoxide (DMSO) purchased from Sigma Aldrich Ltd.

\subsection{Thick film preparation}

PEDOT:PSS/organic solvent solutions were prepared by mixing PEDOT:PSS aqueous dispersion with EC, ES and DMSO. Subsequent depositions by casting of such prepared solutions on polytetrafluoroethyle (PTFE) substrate and solvent removal at elevated temperature for period of $12-48 \mathrm{~h}$ resulted in thick PEDOT:PSS film.

\subsection{Measurement method}

The thickness of PEDOT:PSS films were evaluated by micrometre with accuracy $\pm 1 \mu \mathrm{m}$ (Mitsutoyo Co. Ltd). Electrical resistivity was measured by standard fourprobe method with digital nano-voltometer (Model 2182, Keithley) and dc source meter (Model 2400, Keithley), while the temperature dependence of resistivity with nano-voltometer (Model 34420A, Agilent) and dc source meter (Model 7651, Yokogawa) from 5K to $285 \mathrm{~K}$ using Gifford-McMahon refrigerator system (Model C15PR, ULVAC Cryogenics).

\section{RESULTS AND DISCUSSION}

\subsection{Solvent effect}

The dependence of PEDOT:PSS film prepared from the organic compound added PEDOT:PSS aqueous dispersion (PH grade) on electrical resistivity is shown in Fig. 1. According to Fig.1 the lowest resistivity values for PEDOT:PSS film were observed at room temperature for the $75 \mathrm{mg}$ of EC $(8.3 \mathrm{~m} \Omega \mathrm{cm}), 2.15 \mathrm{~g}$ of ES $(7.2 \mathrm{~m} \Omega \mathrm{cm})$ and $200 \mathrm{mg}$ of DMSO $(3.8 \mathrm{~m} \Omega \mathrm{cm})$ added to $1 \mathrm{~g}$ of PEDOT:PSS dispersion (PH grade). The electrical resistivity of prepared PEDOT:PSS pristine (PH grade) film was $44 \Omega \mathrm{cm}$. Such significant drop of electrical resistivity by three or four orders of magnitude was reported previously for solvents of different nature i.e. polyols [7],[8] and sulfoxides [9]. The cases of EC and DMSO are similar to the previous report using ethylene glycol [10]. The secondary solvent effect for improving the resistivity is not linear with the content but has an optimum value around roughly several to 20 $w t \%$. In contrast, we observed large difference of the optimum content of ES to the other solvents for the minimum resistivity. In the case of ES, almost one order of large volume is necessary in comparison to the cases of EC and DMSO. One of the reasons for such large difference is expected to be degrees of solution of the solvent into the PEDOT:PSS aqueous dispersion. ES is polar aprotic and almost non-miscible with aqueous solutions. This chemical property of ES results in the visible phase separation found in the mixture of PEDOT:PSS/ES, that is, a separation probably between PEDOT:PSS/less-ES dispersion (dark blue colour) and ES-rich solvent (semi-transparent yellow colour) layers. These separations are not observed in the cases of EC and DMSO. The solutions for the film fabrication are extracted from the PEDOT:PSS/ES dispersion layer. Therefore the actual content of ES in the dispersion layer may be rather smaller than the nominal content. The details for the origin of the optimum content of the solvent and the relation to the phase separation are not conclusive at present. Further investigations are necessary.

Based on the recent structural model of ethylene glycol post-treated PEDOT:PSS pristine film [6], this sudden decrease in electrical resistivity can be explained by (1) formation of highly ordered nano-crystalline PEDOT molecules, being part of hydrophobic core zone of PEDOT:PSS particle, (2) thinning of insulating PSS layer between each of PEDOT:PSS particles and

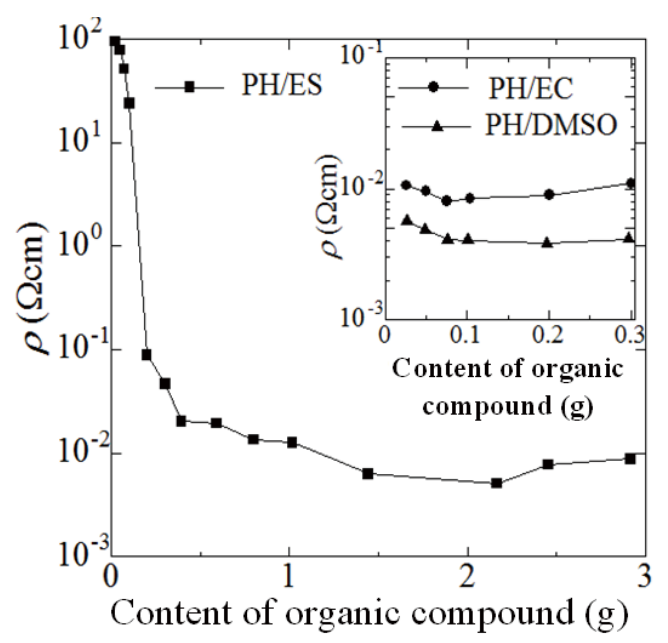

Fig. 1 The electrical resistivity of PEDOT:PSS film (PH grade) prepared from the organic compound added PEDOT:PSS aqueous dispersion of $1 \mathrm{~g}$. 
(3) reinforcement of $\pi-\pi$ interactions. The preparation method of PEDOT:PSS/organic compound added film differs from PEDOT:PSS pristine post-treated film with organic solvents, nevertheless the mechanism of conductivity enhancement can be explained through the same reasoning.

\subsection{DC resistivity}

It is well understood that conductivity enhancement is greatly dependent on structural nature and concentration of organic compound added, therefore an effect on carrier transport properties should be expected; the effect of organic compound on electrical resistivity $\rho$ at RT of organic compound added PEDOT:PSS films is presented in Table I. The values of the dielectric constant of organic compound can be related to the strength of the screening effect between PEDOT polycation which is entangles to PSS poly-anion by strong electrostatic interactions. Ideally, organic compound with larger polarization should have greater effect on the electrical conductivity of PEDOT:PSS film prepared with its addition.

The temperature dependence of electrical resistivity $\rho(T)$ of the PEDOT:PSS follows the variable range hopping (VRH) model [11],

$$
\begin{aligned}
& \rho=\rho_{\mathrm{o}} \exp \left(\frac{T_{\mathrm{o}}}{T}\right)^{\alpha}, \\
& \alpha=\frac{1}{1+D},
\end{aligned}
$$

Table I Chemical structure, dielectric constant $\varepsilon$, dipole moment $\mu$ [12] of added organic compounds and electrical

\begin{tabular}{|c|c|c|c|c|c|}
\hline \multirow{2}{*}{ Name } & \multirow{2}{*}{ Structure } & \multirow[b]{2}{*}{$\varepsilon$} & \multirow{2}{*}{$\mu(\mathrm{D})$} & \multicolumn{2}{|c|}{$\rho(\mathrm{RT})(\mathrm{m} \Omega \mathrm{cm})$} \\
\hline & & & & $\mathrm{PH}$ & PH1000 \\
\hline $\mathrm{EC}$ & & $90^{* * 12 a)}$ & $5.3^{* 12 c)}$ & 8.3 & 3.1 \\
\hline ES & & $39^{* 12 a)}$ & $3.6^{* 12 d)}$ & 7.2 & 2.9 \\
\hline DMSO & & $47^{* * * 12 b)}$ & $3.9^{* 12 b)}$ & 3.8 & 2.8 \\
\hline
\end{tabular}
resistivity $\rho(\mathrm{RT})$ of PEDOT:PSS films.

$*$ at $\mathrm{RT}, * *$ at $40^{\circ} \mathrm{C}$, at $* * * 20^{\circ} \mathrm{C}$ where $D$ is the dimensionality of the electrical conduction path and $T_{\mathrm{o}}$ is interpreted as an effective energy barrier for hopping of carriers between localized states due to disorder. It is described by

$$
T_{o}=\frac{\beta}{N\left(E_{F}\right) \xi^{3} k_{\mathrm{B}}}
$$

where $k_{\mathrm{B}}$ is the Boltzmann constant, $N\left(E_{\mathrm{F}}\right)$ the density of states at the Fermi level, $\xi$ is the localization length and $\beta$ is a numerical factor.

Fig. 2 shows the temperature dependence of the electrical resistivity. The electrical resistivity monotonically increases with temperature approaching to low temperature region. This is indication of semiconductor-like behaviour. It is worth noting that this behaviour diminishes with use of EC and DMSO, however showing still up-curve in low temperature region. This is contrary to PEDOT:PSS/ES case, where at around $60 \mathrm{~K}$ the curvature reinforcement occurs with observable intersection for both PH and PH1000 grades. The electrical resistivity below $60 \mathrm{~K}$ of PEDOT:PSS/ES sample becomes higher than that of PEDOT:PSS/EC, however exhibiting lower electrical resistivity above $60 \mathrm{~K}$.

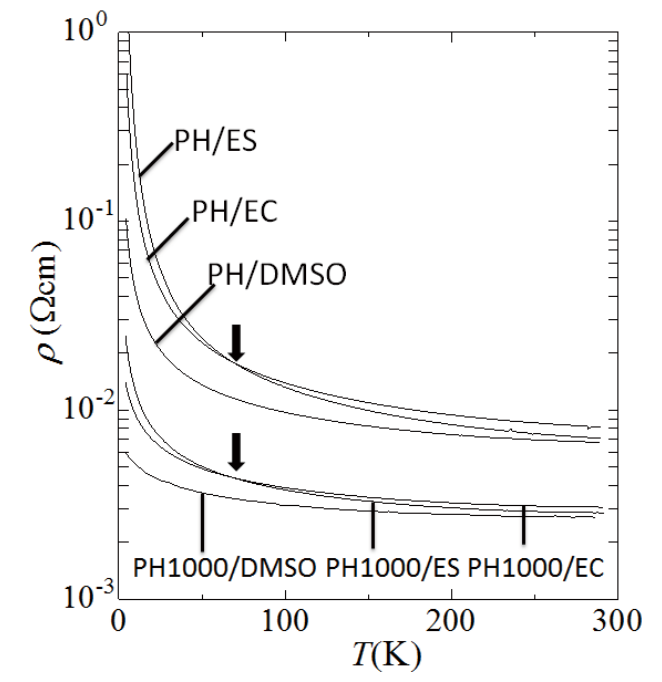

Fig. 2 Temperature dependence of DC resistivity of PEDOT:PSS prepared from different organic solvents. The intersection between PH/ES and EC and $\mathrm{PH} 1000 / \mathrm{ES}$ and EC are indicated by arrows.

Figs. 3 and 4 show the logarithmic plot of the resistivity with the function of $T^{1 / 4}$ in 3D-VRH model for all prepared samples. The dashed straight lines in 
Figs. 3 and 4 demonstrate that the electronic conduction follows well Eqs. (1) and (2) with $D=3$ at low temperatures below approximately $20 \mathrm{~K}$. Although we tried to apply the other dimensionality of $D=2$ and 1 , and also the thermal activation-type behaviour, including small polaron conduction, the present 3DVRH model gives the best result for the fittings at low temperatures. At higher temperatures, however, the resistivity does not follow simple numerical expression like as VRH model with fixed $\alpha$ value. It is noted here that the electronic conduction mechanism should be evaluated at low enough temperatures at which other material properties like as thermal contraction become temperature independent. The parameter $T_{0}$ has a tendency to approach lower values with decreasing resistivity for ES, EC and DMSO added PEDOT:PSS films with the change of the grade, therefore indicating lower effective energy barrier and longer localization length of carrier charges. This is in accordance with the VRH model claiming that the localization length $\xi$ will increase as resistivity decrease. Although the general tendency and relation between $T_{0}$ and the behaviour of the resistivity in all samples are fairly well understood in the framework of VRH model, the obtained small value of $T_{0}$ in the lowest resistivity sample, PH1000/DMSO, suggests that the weak increase of the resistivity for temperature may happen to coincident with 3D-VRH model. Because the temperature region for the VRH evaluation should be lower than the value of $T_{0}$. But the resultant value of $T_{0}=1.3 \mathrm{~K}$ does not satisfy the condition. Therefore, the fairly low resistivity sample with the order of $\sim \mathrm{m} \Omega \mathrm{cm}$ may not possess a carrier

Table II Electrical resistivity $\rho(\mathrm{RT})$, effective energy barrier between localize states $\left(T_{\mathrm{o}}\right)$ and infinite temperature resistivity $\rho_{\mathrm{o}}$ of PEDOT:PSS/organic compound film.

\begin{tabular}{cccc}
\hline Sample & $\begin{array}{c}\rho(\mathrm{RT}) \\
(\mathrm{m} \Omega \mathrm{cm})\end{array}$ & $T_{\mathrm{o}}(\mathrm{K})$ & $\rho_{\mathrm{o}}(\Omega \mathrm{cm})$ \\
\hline $\mathrm{PH} / \mathrm{EC}$ & 8.3 & 19200 & 0.19 \\
$\mathrm{PH} / \mathrm{ES}$ & 7.2 & 78100 & 0.02 \\
$\mathrm{PH} / \mathrm{DMSO}$ & 3.8 & 1930 & 1.05 \\
$\mathrm{PH} 1000 / \mathrm{EC}$ & 3.1 & 51.1 & 2.09 \\
$\mathrm{PH} 1000 / \mathrm{ES}$ & 2.9 & 552 & 0.82 \\
$\mathrm{PH} 1000 / \mathrm{DMSO}$ & 2.8 & 1.3 & 2.91 \\
\hline
\end{tabular}

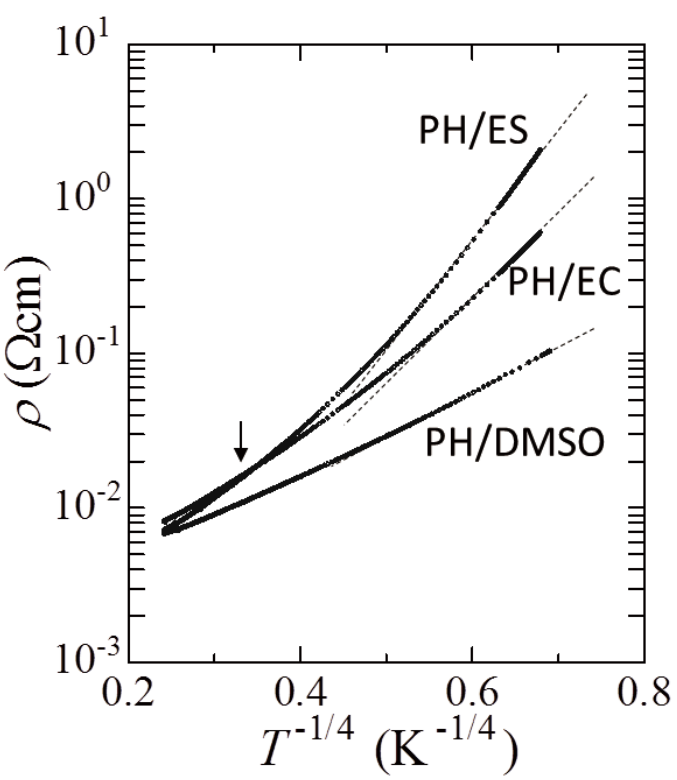

Fig. 3 Temperature dependence of DC resistivity of PEDOT:PSS films for PH/ES, EC and DMSO. The dashed straight lines indicate that the resistivity follows the 3D VRH model at low temperatures.

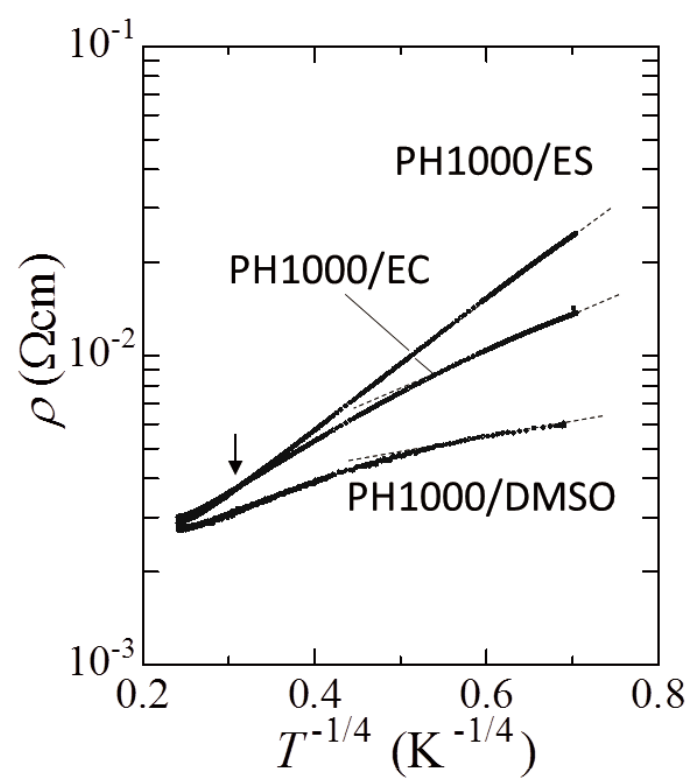

Fig. 4 Temperature dependence of DC resistivity of PEDOT:PSS films for PH1000/ES, EC and DMSO. The dashed straight lines indicate that the resistivity follows the 3D VRH model at low temperatures. The low resistivity and small slope of the straight line in PH1000/DMSO indicate a metallic tendency of the electronic conduction rather than the electron hopping. The details see the text.

hopping conduction but a metallic conduction. In very recent study, a solvent post treated PEDOT:PSS sample 
shows a metallic temperature dependence [13]. We need to consider a crossover from VRH to metallic conduction in further research on the mechanism of the charge transport and the improvement of the conductivity.

\section{CONCLUSION}

We have shown that utilization of secondary dopants such as EC and ES for PH1000 garde lowers the electrical resistivity significantly, reaching $3.1 \mathrm{~m} \Omega \mathrm{cm}$ and $2.9 \mathrm{~m} \Omega \mathrm{cm}$, respectively. We observe gentle disappearance of semiconducting like-behaviour by upcurve reduction in the low temperature region $\mathrm{T}<10 \mathrm{~K}$. Temperature dependence of the electrical resistivity of PEDOT:PSS/EC and PEDOT:PSS/ES films are in agreement with 3D-VRH model. On the other hand, a metallic transport mechanism should be considered in the lower resistivity sample at low temperature.

\section{ACKNOWLEDGMENT}

This work is partly supported by Grants for Excellent Graduate Schools, MEXT, Japan and the Industry-Academic Collaborative R\&D of Japan Science Technology Agency.

\section{REFERENCES}

[1] A. Elschner, S. Kirchmeyer, W. Lovenich, K. Reuter and U. Merker, "PEDOT", ed. 1, CRC Press, Boca Raton (2010), p. 167-243.

[2] L. Groenendaal, G. Zotti and F. Jonas, Synth. Met., 118, 105-109 (2001).

[3] P. A. Levermore, L. C. Chen, X. H. Wang, R. Das and D. D. C. Bradley, Adv. Mater., 19, 2379-2385 (2007).

[4] Y. H. Kim, C. Sachse, M. L. Machala, C. May, L. M. Meskamp and K. Leo, Adv. Funct. Mater., 21, 10761081 (2011).

[5] A. G. MacDiarmid and A. J. Epstein., Synth. Met., 65, 103-106 (1994).

[6] T.Takano, H. Masunaga, A. Fujiwara, H. Okuzaki and T. Sasaki, Macromolecules, 45, 3859-3865 (2012)

[7] B. D. Martin, N. Nikolov, S. K. Pollack, A. Saprigin, R. Shashidhar, F. Zhang and P. A. Heiney, Synth. Met., 142, 187-193 (2004).
[8] S. K. M Jönsson, J. Birgerson, X. Crispin, G. Greczynski, W. Osikowicz, A.W. Denier van der Gon, W. R. Salaneck and M. Fahlman, Synth. Met., 139, 1-10 (2003).

[9] J. Ouyang, Q. Xu, C. W. Chu, Y. Yang, G. Li and J. Shinar, Polymer, 45, 8443-8450 (2004).

[10] X. Crispin, F. L. E. Jakobsson, A. Crispin, P. C. M. Grim, P. Andersson, A. Volodin, C. van Haesendonck, M. Van der Auweraer, W. R. Salaneck and M. Berggren $\dagger$, Chem. Matte., 18, 4354-4360 (2006). [11] N. F. Mott and E. Davis, "Electronic Processes in Non-Crystalline Materials", ed. 2, Oxford University Press, Oxford (1979), p. 32-37.

[12] a) Ch. Wohlfahrt, "2 Pure Liquids: Data, The Landolt-Börnstein Database", O. Madelung (ed.), Springer Materials, www.springermaterials.com; b) www.sigmaaldrich.com/chemistry/solvents/dimethylsulf oxide-center.html; c) J. L. Alonso, R. Cervellati, A. D. Esposti, D. G. Lister and P. Palmieri, J. Chem. Soc., Faraday Trans., 2, 357-366 (1986); d) D. S. Breslow and H. Skolnik, "The Chemistry of Heterocyclic Compounds", Part 1, ed. 1, Interscience, New York (1966), p. 5.

[13] Y. Honma, T. Nishizaki, M. A. Guziak and T. Sasaki, (unpublished), presented at 68th Annual meeting of the Physical Society of Japan, 27pXP-1 (2013).

(Received February 27, 2013; Accepted May 1, 2013) 